\title{
Adverse reactions to food additives
}

\author{
By Barbara MacGibbon, Department of Health and Social Security, Hannibal \\ House, Elephant and Castle, London $S E_{1} 6 T E$
}

The first published report of an adverse reaction to a food additive was that provided by Lockey (1959). He described urticaria in a patient who was given steroid therapy, the formulation of which contained tartrazine. In 1973 the Ministry of Agriculture, Fisheries and Food's Food Additives and Contaminants Committee produced an interim report on the Review of the Colouring Matter in Food Regulations. In the report the Committee on Toxicity of Chemicals in Food, Consumer Products and the Environment noted that there had been a number of reports of hypersensitivity reactions following the experimental ingestion of certain food colours; the evidence suggested that such reactions were rare. The Committee further noted that there was suggestive evidence of idiosyncratic reactions following ingestion of food containing usual levels of colouring. They were concerned about the situation but as hypersensitivity to food constitutes a general problem and is not confined to colours, they felt that useful recommendations could not be made until clearer evidence from well-controlled studies in man became available. In 1979 the Commission of the European Community's Scientific Committee for Food reported on certain colouring matters and noted similar problems.

It is obviously easier to detect the presence of colouring matter than other additives in foods and there can be no doubt that this situation has led to colours being tested more frequently than other additives for their potential to produce adverse reactions in sensitive individuals. Furthermore, the reasons for using colouring matters (for example, in food to replace colour lost in processing, to inhibit or mask fading, and to ensure uniformity in the appearance of a particular product; and in medicines to aid correct identification) are not universally accepted. The Scientific Committee for Food, knowing of concern about the use of colouring matters in food, were also aware of the possibility that other food additives could provoke adverse reactions. The Committee therefore suggested to the Commission, in 1979, that a working group should be set up to consider adverse reactions to ingested additives present in food, drug formulations and cosmetics. This working group presented its findings to the Scientific Committee for Food in $198 \mathrm{I}$ and its report was published the following year (Commission of the European Community, I 982). Much of the following presentation will be based on participation in this working group, but an attempt will be made to emphasize those questions of particular concern, from a regulatory point of view, for the UK. 


\section{What is the extent of the problem?}

The main tasks identified by the working group were to review the available evidence in order to define the size of the problem and to establish a scientific basis for recommending what should be done in terms of research as well as regulatory action. It was not only necessary to reach some conclusion about the frequency of adverse reactions attributable to ingestion of additives, but to gain some idea of the extent of the problem also in terms of whether few or many additives were involved and the range of clinical manifestations and their severity. One of the difficulties in arriving at an estimate of the frequency of adverse reactions is that of finding reliable evidence associating an adverse reaction with the ingestion of an additive. Such adverse reactions are clinically indistinguishable from allergic responses to a variety of foods. Even in the latter case there may be difficulty in relating symptoms to food ingestion, particularly if the onset of symptoms is delayed or they are non-specific in type. It is even more difficult to identify a particular additive in such a causal relationship, especially since most of the reactions have not as yet been shown to be immunologically determined and there is, therefore, a lack of specific or useful diagnostic tests such as the skin test, or radio-allergosorbent test for specific IgE antibodies. The existing means of determining the relationship between an additive and an adverse reaction are time consuming and include history taking and observations on avoidance diets and after challenge testing. Unequivocal evidence of such an effect requires doubleblind challenge testing with placebo on more than one occasion. Results from such studies are not readily available and most of the data considered are less satisfactory.

In considering the types of clinical manifestation which may result from adverse reactions to food additives, it is clear that it is easier to obtain evidence in patients whose respiratory or skin problems are aggravated by ingestion of particular additives, than to suspect that rather non-specific gastrointestinal symptoms, for example, may be produced in a similar way. There is better evidence relating these three types of symptom to ingestion of additives than is the case with neurobehavioural problems. Nevertheless, the suggestion that food additives could cause neurobehavioural problems is worth considering in some detail.

\section{Behavioural effects}

Feingold ( 1973 ) first proposed an association between ingestion of food additives and behavioural problems. He noted in some of his adult allergic patients, who showed sensitivity to aspirin, that there was a behavioural element involved in their symptoms. This observation led him to suggest, initially, that naturally occurring salicylates should be excluded from the diets of children showing hyperactive behaviour. He subsequently suggested, on the basis of the known cross reactivity between tartrazine and aspirin in sensitive subjects, that tartrazine should also be excluded. The next step was to exclude other colouring matters from the diets and finally artificial flavours too. The particular group of patients in whom the Feingold exclusion diet has been extensively tried is that of hyperactive children. One of the problems in assessing the usefulness of the Feingold regimen 
in such children is that hyperactivity is not a single disease entity but a syndrome of purposeless over-activity, lack of concentration and resultant learning difficulties, which can result from a variety of causes. This problem of identifying a homogeneous group of children, with unequivocally the same disorder, is reflected in the different figures for frequency obtained in the UK and the United States, the figures being $0.09 \%$ and from I.I 9 to $5 \%$ respectively. A joint study is currently underway to determine the criteria for diagnosing this condition in the UK and in the United States.

Additional problems in evaluating the effectiveness of Feingold's diet result from inconsistencies or inadequacies in the known composition of his exclusion diets. For instance, there are inconsistencies in the salicylate levels of foods which are included or excluded; some artificial flavours (excluded) are identical to those found in nature and it is not possible to deduce which additives could be held responsible for hyperactivity. Feingold claimed a $50 \%$ success rate on the basis of case histories, but this has not been substantiated by double-blind trials using placebo diets or bolus challenges. In some such controlled studies children were selected according to defined behavioural criteria, or particular target symptoms were identified, and assessments were carried out by independent observers, teachers and/or parents. Under these circumstances, colours (blended according to their use in food, and used up to a calculated total daily intake dose of 27 or $3^{6} \mathrm{mg}$ ) provided no confirmation of Feingold's claims. Even bolus doses of 100 or $150 \mathrm{mg}$ of blended colours, when given to children who had reputedly responded to a Feingold diet, did not produce an exacerbation of their hyperactivity. However, in two of these studies (Connors et al. 1976; Swanson \& Kinsbourne, 1980) it was suggested that there was evidence of some impairment of the children's ability to perform laboratory tests, such as visual tracking performance or paired associate learning; these studies require confirmation.

From animal experiments there is some evidence of behavioural effects, at an equivalent dosage to a child's intake of food colours, on the activity and habituation of rat pups. However, this effect did not show a dose-response relationship (Shaywitz et al. 1978). One colour, erythrosine, has been specifically tested in vitro and shown to inhibit the uptake of neurotransmitters by the nerve cell (Lafferman \& Silbergeld, 1979; Logan \& Swanson, 1979). Different results have, however, been obtained in different experimental conditions and erythrosine has not shown any effect on activity even in dopamine-deficient rats which are a possible model of hyperactivity (Mailman et al. 1980). Neither has erythrosine produced any impairment of paired associate learning in hyperactive children at doses of $\mathrm{r} \cdot 3 \mathrm{mg} / \mathrm{kg}$ (J. M. Swanson, unpublished results).

In conclusion, it would be reasonable to state that no evidence in support of Feingold's hypothesis has been derived from properly controlled studies. However, it should be noted that because of technical difficulties in disguising flavours in placebo and challenge food, similar studies have not been carried out for this type of food additive. It is therefore advisable to reserve judgement on the possible behavioural effects of some colours. 


\section{Frequency of adverse reactions}

Returning now to the question of frequency of adverse reactions to food additives it is worthwhile noting, as background information, estimates of the prevalence of food allergy. It is calculated that in Denmark not less than $0.2 \%$ of the population suffers from food allergy (Poulsen, 1980) and the comparable figures for France are $0.5^{-1.0 \%}$ (Moneret-Vautrin \& Grilliat, 1979), and for the USA $<\mathrm{r} \%$ (Smith, 1974). It is not intended to suggest that any problem of less magnitude is unimportant but merely to provide a context in which to consider the problem of adverse reactions due to food additives.

We have no real knowledge about the frequency of adverse reaction to food additives in the general population. Such estimates as are available are based on challenge testing in highly selected population groups. The calculation is made from the basis of establishing the percentage of patients with a condition such as chronic urticaria who react to a particular additive; then considering the percentage of the general population who are thought to suffer from chronic urticaria and, finally, deriving the percentage of the general population who may be expected to be sensitive to the additive in question. Thus the frequency of reactions to tartrazine and benzoate have been calculated as $0.1 \%$ of the population (Poulsen, 1980). The validity of such data can be questioned from several points of view. First, the percentage of the patients giving a positive reaction may depend on such factors as the dose used in testing, the time of measuring response, the phase of the disease, the state of the gastrointestinal tract at the time and the type of challenge test used, e.g. whether this was repeated on more than one occasion, whether an open trial was conducted or a single- or doubleblind study. Second, this procedure assumes that patients with urticaria, for example, are no more likely to show sensitivity to additives than normal subjects and that no other group of patients would show a greater incidence of sensitivity.

Table I. Results obtained by challenge testing with different additives in patients with chronic urticaria

$\begin{array}{lcc}\text { Challenge } & \% \text { Positive reactions } & \text { Reference } \\ \text { Aspirin } & 22 & \text { Juhlin, 198I } \\ \text { Azo dyes } & 18 & \\ \text { Benzoates } & 11 & \\ \text { Annatto } & 10 & \\ \text { BHA, BHT } & 15 & \\ \text { Quinoline yellow } & 13 & \\ \text { Yeast } & 16 & \text { Mikkelson et al. } 1978 \\ \text { Annatto } & 26 & \\ \text { Tartrazine } & 11 & \\ \text { Sunset yellow } & 17 & \\ \text { Food red } 17 & 16 & \\ \text { Amaranth } & 9 & \\ \text { Ponceau } 4 \mathrm{R} & 15 & \\ \text { Erythrosine } & 12 & \\ \text { Brilliant blue } & 14 & \end{array}$


While this is probably true for patients with respiratory tract disorders, such as asthma, there is inadequate information on which to judge the situation among patients with gastrointestinal manifestations. On the basis of this kind of calculation it is possible that between 0.03 and $0.15 \%$ of the population is sensitive to tartrazine. This would suggest something of the order of a tenfold difference between the occurrence of general food allergies and those attributable to food additives such as tartrazine.

\section{Are certain additives more harmful than others?}

Another question which was considered important from a regulatory point of view was whether some additives are more capable of provoking adverse reactions than others. The evidence consists of the results of challenge studies carried out in patients with disorders such as chronic urticaria, or asthma, using a variety of additives. Obviously, the limitations already mentioned in the use of this kind of challenge study apply equally to making comparisons between additives. But the evidence shown in Table $I$ indicates that artificial colours in general, and tartrazine in particular, are not alone among food additives in their ability to provoke adverse reactions in some patients under particular conditions of testing. Some of the notoriety of tartrazine must be attributed to the fact that it is very widely and obviously used, and frequently tested. On the question of whether particular additives are likely to cause more severe reactions of an anaphylactic type, there is very little information. Anaphylactic reactions attributed to ingestion of additives have been documented more rarely than those attributed to food, but tartrazine, sunset yellow, ponceau red and quinine have been implicated.

\section{Other questions of regulatory concern}

The working group also looked for information about whether children were more susceptible to adverse reactions after ingestion of additives than adults. On the basis of results obtained from challenge testing in children and adults who suffer from asthma, it seems that, unlike the situation with food allergy, children are not consistently more susceptible than adults to adverse effects from additives (Table 2).

Another question which was considered was whether there was any evidence to suggest that food additives were capable of causing a disease, such as asthma or chronic urticaria, rather than provoking or aggravating a pre-existing condition because of some underlying sensitivity. It was felt by the working group that any evidence that an additive such as tartrazine could initiate a disease process would call for a ban; no such evidence was found. It is not known whether a defect resulting in a particular susceptibility is genetically determined in sensitive subjects or is acquired.

\section{The mechanism(s) of production of adverse effects following ingestion of additives}

It has been mentioned already that the clinical effects in adverse reactions to food or food additives are similar, whether the specific trigger for histamine and other mediator release is immunologically determined or due to some idiosyncracy. 
Table 2. A comparison of results obtained on challenge testing adult and juvenile asthmatics with food additives

\begin{tabular}{|c|c|c|}
\hline Challenge & $\%$ Positive results & Reference \\
\hline \multicolumn{3}{|l|}{ Adult asthmatics } \\
\hline Aspirin & 8-10 & \multirow{3}{*}{$\begin{array}{l}\text { Settipane et al. 1974; } \\
\text { Settipane \& Pudupakkam, 1975; } \\
\text { Juhlin et al. 1972 }\end{array}$} \\
\hline (in severe ast & natics) I6-1 8 & \\
\hline Cross-reaction with tartrazine/benzoates & $8-15$ & \\
\hline \multicolumn{3}{|l|}{ Juvenile asthmatics } \\
\hline Aspirin & 33 & Syvanen \& Backman, 1978 \\
\hline Sodium benzoate & 18 & \\
\hline Tartrazine & 20 & \\
\hline \multicolumn{3}{|l|}{ Aspirin, benzoates, dyes } \\
\hline $\begin{array}{l}\text { on initial testing } \\
\text { double-blind retesting with placebo }\end{array}$ & $\begin{array}{r}24 \\
6.5\end{array}$ & Østerballe et al. 1979 \\
\hline Tartrazine & 0 & Vendanthan et al. 1977 \\
\hline
\end{tabular}

The need to understand the mechanism whereby food additives can provoke adverse reactions lies not only in the desirability of having a diagnostic test, but also in the provision of more accurate means of conducting epidemiological studies in the general population than history taking and challenge testing. Equally important is the need to understand the mechanism of these adverse reactions for the development of models for predictive toxicity testing in new food additives. Table 3 outlines the possible mechanisms for production of adverse reactions. The fact that food additives are small molecular weight chemicals would require hapten formation with in vivo binding to protein, for the production of an immunological response. In the case of tartrazine, for example, there is little evidence of such in vivo binding. It may be that metabolites or impurities are the active agents in some circumstances. There are sufficient results available to suggest that the classical type I response mediated through IgE-specific antibodies is not responsible for the adverse reactions provoked by food additives. There is some evidence that IgD-specific antibody may be of importance (Weliky et al. 1979). At the moment there are insufficient results available on which to assess the possibility of immune-complex formation being involved.

\section{Table 3. Mechanism(s) of production of adverse reactions to food additives}

1. Immunological (haptens formed in vivo)

IgE mediated, type I, immediate hypersensitivity

$\operatorname{Ig} \mathrm{D}$

type III Immune-complex disease

type IV delayed cellular hypersensitivity

2. 'Pharmacological'

Inhibition of prostaglandin synthesis (cf. aspirin cross-reactivity)

Idiosyncratic

3. Influence of gastrointestinal tract conditions

4. Problems due to metabolites or impurities? 
Delayed hypersensitivity types of reaction have been recorded. For example eczema or contact dermatitis has, rarely, followed the ingestion of benzoates, azo dyes or antioxidants to which the subject had previously been exposed in cosmetic preparations or topical medicinal products. In terms of pharmacological or idiosyncratic mechanisms it should be noted that the possibility of tartrazine interfering with prostaglandin synthesis, because of its cross reactivity with aspirin, has been investigated without producing satisfactory evidence for this mode of action.

Little work has been carried out on possible idiosyncratic mechanisms associated with specific enzyme defects, which would enhance mediator release or activity; nor is much known about the possibility of liver enzyme abnormalities affecting the metabolism of suspect additives. However, one important factor contributing to the ability of additives to cause adverse reaction in certain individuals is undoubtedly the integrity of the gastrointestinal tract. Anything which predisposes to the increased absorption of an additive will enhance its ability to cause an adverse reaction. For instance, if the level of IgA is reduced, the permeability of the gut wall increased or the activity of bacterial flora changed, the absorption of the additive or a metabolite may be enhanced. It is therefore important to take account of antibiotic or other therapies, alcohol consumption and the composition of the diet as well as any co-existing gastrointestinal disorders, in considering adverse reactions.

\section{Research needed}

The two major gaps in our knowledge, identified by the working group, concerned proper results on the extent of the problem of adverse reactions to ingested additives in the general population, and understanding of the mechanism(s) for the production of these reactions. Although epidemiological studies on randomly selected groups from the general population would be facilitated by the availability of a reliable diagnostic test, this is not an essential requirement. Information on the incidence and prevalence of adverse reactions could be obtained by means of careful history taking and challenge testing of individuals. Information which might throw some light on the mechanism(s) of production of adverse reactions could be obtained by studying differences in the genetic, immunological and pharmacological background of reactors and of non-reactors (gastrointestinal factors would obviously be of importance too). Finally, the detailed study of reactors under challenge conditions would hopefully reveal the mechanism involved in the release of mediators. Without this understanding of what happens in sensitive human subjects it may not prove possible to develop animal or in vitro models for predictive testing of new additives.

\section{Proposals for regulatory action}

The remaining objective of the working group was to define the scientific basis on which recommendations could be offered for regulatory action. There is clear 
evidence of the existence of a problem, possibly affecting I in 1000 of the population and involving a variety of additives. The majority of the adverse reactions produced are not severe and there is some evidence from challenge studies of the existence of a threshold effect. While it was considered that there was insufficient evidence to support a ban on the use of tartrazine (or all azo dyes, or even all colours), it was recommended that the use of colours in food should be reduced, and that specific labelling be introduced to identify the presence of all additives in foods and so make it easier for sensitive subjects to avoid them. The situation at the moment in the European Community is that a Directive was issued at the end of 1978 , requiring specific labelling. However, a derogation, taken up by the UK, allowed generic labelling until 1983. The Directive has not yet been revised to create common rules for member states. However, the Ministry of Agriculture, Fisheries and Food, following the endorsement of the European Community's working group report by the Committee on Toxicity and the Food Additives and Contaminants Committee, is shortly to release proposals for specific labelling in the UK by January 1985 .

\section{REFERENCES}

Commission of the European Community (1982). Food-Science and Techniques. Reports of the Scientific Committee for Food (1 2th series), EUR 7823.

Connors, C. K., Goyette, C. H., Southwick, D. A., Lees, J. M. \& Andrulonis, P. A. (1976). Paediatrics 58, 154.

Feingold, B. F. (1973). Hosp. Prac. 8, I I, i 7, 2 I.

Juhlin, L. (1981). Br. J. Derm. 104.

Juhlin, L., Michaelsson, G. \& Zetterstrom, O. (1972). F. Allerg. Clin. Immunol. 50, 92.

Lafferman, J. A. \& Silbergeld, E. K. (1979). Science, 205, 4 10.

Lockey, S. D. (1959). Ann. Allergy 17, 719.

Logan, W. J. \& Swanson, J. M. (1979). Science 206, 363.

Mailman, R. B., Ferris, R. N., Tang, F. L. M., Vogel, R. A., Kilts, C. D., Lipton, M. A., Smith, D. A., Mueller, R. A. \& Breese, G. R. (1980). Science 207, 535.

Mikkelson, H., Larsen, J. C. \& Tarding, F. (1978). Arch. Toxicol. Suppl. 1, 14 I.

Moneret-Vautrin, D. A. \& Grilliat, J. P. (1979). Méd. Nutr. 15, 301.

Østerballe, O., Taudorf, E. \& Haahr, J. (1979). Ugeskr. Laeg. 141, I 908.

Poulsen, E. (1980). Danish report on allergy and intolerance to food ingredients and food additives. Toxicology Forum, Aspen, Colorado.

Settipane, G. A., Chafee, F. H. \& Klein, D. E. (1974). J. Allergy Clin. Immunol. 53, 200.

Settipane, G. A. \& Pudupakkam, R. K. (1975). F. Allergy Clin. Immunol. 56, 2 I 5.

Shaywitz, B. A., Goldenring, J. R. \& Wool, R. S. (1978). 7th Annual Child Neurology Society Meeting, Keystone, Colorado.

Smith, J. M. (1974). Med. Clins. N. Am. 58, 3.

Swanson, J. M. \& Kinsbourne, M. (1980). Science 207, 1485.

Syvanen, P. \& Backman, A. (1978). Abstracts, Nordic Congress of Allergology, Copenhagen, p. 58.

Vandanthan, P. K., Menon, M. M., Bell, T. D. \& Bergin, D. (1977). F. Allergy Clin. Immunol. $60,8$.

Weliky, N., Heiner, D. C., Tamura, H., Anderson, S., Stenius-Aarniala, B., German, D., Hawley, C. D. \& Lockey, S. D., Sr (1979). Immunol. Commun. 8, 65. 